\title{
Origin of Neutrino Mass
}

\section{Goran Senjanović}

Gran Sasso Science Institute, Viale Crispi, L'Aquila, Italy International Centre for Theoretical Physics, Trieste, Italy

E-mail: goranegssi.infn.it

\section{Vladimir Tello}

Gran Sasso Science Institute, Viale Crispi, L'Aquila, Italy

E-mail: tello@gsi.infn.it

There are strong indications that charged fermions masses have origin in the Higgs mechanism. We review here attempts that provide the same source for neutrino mass. If one sticks to simple scenarios, the type II seesaw comes closest to achieving this, whereas if one looks for more complete theories the minimal LR symmetric model stands out as the theory that led originally to neutrino mass, the seesaw mechanism and the lepton number violation at hadronic colliders. These historical developments are deeply connected with the neutrinoless double beta decay and a plethora of low energy lepton number and flavor violating processes. Moreover, the theory offers a potential LHC probe of the Higgs origin of neutrino mass in analogy with charged fermions. We offer here a short review of these issues. The examples of other well motivated theories are the MSSM and the minimal extension of the minimal $S U(5)$ grand unified theory, discussed en passant.

18th International Conference From the Planck Scale to the Electroweak Scale 25-29 May 2015

Ioannina, Greece 


\section{Introduction}

Now that the Higgs boson has been found, we have a real possibility of probing the origin of mass of elementary particles. The main virtue of the Higgs mechanism is that the Higgs boson decay rates in the Standard Model are completely determined by the masses of particles in question. In particular, the one-to-one correspondence between masses and Yukawa couplings of charged fermions allows one to predict the Higgs boson decays into fermion anti-fermion pairs

$$
\Gamma(h \rightarrow f \bar{f}) \propto G_{F} m_{h} m_{f}^{2} .
$$

The SM fortunately has one failure as we all know: in its minimal version it predicts massless neutrinos, and thus provides a window into new physics. If one wishes to probe the Higgs origin of neutrino mass, one has to do what Weinberg [1] did for charged fermions. We discuss here under which circumstances this may be possible. We go first through simplest possibilities of (i) neutrino being Dirac particle, (ii) neutrino being Majorana particle through the effective $d=5$ approach and (iii) the three seesaw mechanisms.

These scenarios can account a posteriori for neutrino mass. However, neutrino mass was predicted long before experiment by the minimal LR symmetric theory [2], introduced originally in order to account for violation of parity in weak interactions. Moreover, with the advent of the seesaw mechanism [3], neutrino ended up naturally much lighter than the electron. The essential point of the seesaw is that it leads to neutrino being a Majorana particle which implies $\Delta L=2$ violation of lepton number through

a) neutrinoless double beta decay $(0 v 2 \beta)$, suggested [4] soon after Majorana classic work [5]. The LR symmetric theory implies its possible new origin [6] through the Right-Handed (RH) currents, as a clear example of new physics contribution to this process [7].

b) production of same sign charged lepton pairs in hadron colliders (the KS process) [8]. This high energy analog of neutrinoless double beta decay offers the possibility of probing directly the Majorana neutrino nature.

While the neutrinoless double beta decay has been considered a text-book probe of Majorana neutrino mass, the like-sign lepton pair production at hadronic colliders has gained wide attention with arrival of the LHC. Ironically, the LHC could shed more light on neutrino mass than neutrinoless double beta decay itself, since the latter process could be induced by new physics. If the new physics is due to the RH neutrino and gauge boson as in [8], the LHC can in principle test the Higgs mechanism origin of neutrino mass [9], in a similar manner to what the SM does for charged fermions. In order for this to work it is essential to know the RH quark mixing matrix; its analytic form being only recently obtained [17]. These new results arguably show that the LR model is a self-contained and predictive theory of neutrino mass.

What about other theories of neutrino mass potentially manifestable at the LHC? An example is provided by the minimal extension of the minimal $S U(5)$ grand unified theory that leads to the hybrid type I and type III seesaw, and predicts a triplet of fermions in the $\mathrm{TeV}$ regime potentially accessible to the LHC [10]. A more popular example is the Minimal Supersymmetric Standard Model (MSSM) without ad-hoc R-parity conservation; however a huge number of parameters of the MSSM makes it hard, if possible at all, to make precise physical statements. We come back to it in the Summary and Outlook section. 


\section{SM seesaw scenarios and the origin of neutrino mass}

Dirac neutrino. Let us assume first that neutrino is the Dirac particle through the addition of the RH neutrino. In complete analogy with charged fermions, we would have for its Yukawa coupling

$$
Y_{v}^{D}=\frac{g}{2} \frac{m_{v}}{M_{W}}
$$

too small to be ever observed. This could be called a devilish conspiracy where neutrino mass is only visible in neutrino oscillations. It requires however an artificial assumption of practically zero RH neutrino mass, equivalent to ad hoc global lepton number conservation.

Majorana neutrino. If we wish to account for tiny neutrino masses with only the SM degrees of freedom, we need $d=5$ effective operator [11]

$$
\mathscr{L}=c_{i j} \frac{L_{i} H H L_{j}}{M}
$$

where $L_{i}$ stands for left-handed leptonic doublets and $\mathrm{H}$ for the usual Higgs doublet. This in turn produces neutrino Majorana masses. Exactly in the same manner as in the Dirac case, one would obtain for the effective Yukawa coupling, now of Majorana nature

$$
Y_{v}^{M}=\frac{g}{2} \frac{m_{v}}{M_{W}}
$$

equally small and unobservable as in the pure Dirac case.

By itself, the effective $d=5$ interaction offers basically no new accessible physics besides nonvanishing neutrino mass. and so it cries for the UV completion. The simplest one is provided by the seesaw mechanism [3], today considered the main paradigm behind the smallness of neutrino mass. In its simple version where one adds just one type of particles, there are only three seesaw types. One can give up on this assumption, but then one opens a Pandora box of innumerable ways of obtaining neutrino mass, something we will shy away from here.

Type I seesaw. This original seesaw scenario [3], with RH neutrinos $N$ on top of the SM, comes short of doing the job. Even the complete knowledge of $M_{v}$ and $M_{N}$ does not suffice to predict Dirac Yukawa (and associated $N$ decay rates); its determination is plagued by the arbitrary complex orthogonal matrix $O$ [14] whose elements have no upper limit at all.

Type II seesaw. Instead of RH neutrinos, one adds a complex scalar triplet coupled to leptons [12]. Here the situation is much more promising: the knowledge of neutrino masses and mixings allows to predict decay rates of double charged scalars into lepton pairs [15]. The setback is the lack of deeper motivation for the model.

Type III seesaw. This is similar to the type I situation, since one trades singlet RH neutrinos for the $S U(2)$ fermion triplets [13]. Once again there is a problem of an arbitrary orthogonal matrix $O$. 


\section{Left-right symmetry and the origin of neutrino mass}

The idea of LR symmetry comes as a desire to understand the origin of parity violation in weak interactions. It is important to recall that a wish to have parity as a fundamental symmetry in beta decay is as old as the suggestion of its breakdown. In their classic paper, Lee and Yang [18] argue in favor of the existence of the opposite chirality heavy proton and neutron, which would make the world parity symmetric at high energies.

Left-right gauge theory. The LR symmetric gauge theories, on the other hand, keep the fermionic content of the SM intact, and instead doubles the weak gauge sector. The minimal model [2] is based on the following electroweak gauge group

$$
S U(2)_{L} \times S U(2)_{R} \times U(1)_{B-L},
$$

plus a symmetry between the left and right sectors. Quarks and leptons are completely LR symmetric

$$
Q_{L, R}=\left(\begin{array}{l}
u \\
d
\end{array}\right)_{L, R}, \quad \ell_{L, R}=\left(\begin{array}{l}
v \\
e
\end{array}\right)_{L, R} .
$$

The formula for the electromagnetic charge becomes

$$
Q_{e m}=I_{3 L}+I_{3 R}+\frac{B-L}{2} .
$$

LR symmetries. It is easy to verify that the only realistic discrete symmetries exchanging the left and right sectors, preserving the kinetic terms are

$$
P:\left\{\begin{array}{l}
f_{L} \leftrightarrow f_{R} \\
\Phi \leftrightarrow \Phi^{\dagger} \\
\Delta_{L} \leftrightarrow \Delta_{R}
\end{array} \quad C:\left\{\begin{array}{l}
f_{L} \leftrightarrow\left(f_{R}\right)^{c} \\
\Phi \leftrightarrow \Phi^{T} \\
\Delta_{L} \leftrightarrow \Delta_{R}^{*}
\end{array}\right.\right.
$$

where $\left(f_{R}\right)^{c}=C \gamma_{0} f_{R}^{*}$ is the charge-conjugate spinor. The names of $P$ and $C$ are motivated by the fact that they are directly related to parity and charge conjugation, supplemented by the exchange of the left and right $S U(2)$ gauge groups, as is evident from (3.4).

The modern day version of the theory is based on the seesaw mechanism. The Higgs sector consists of the following multiplets [19]: the bi-doublet $\Phi$ and the $S U(2)_{L, R}$ triplets $\Delta_{L, R}$

$$
\Phi=\left[\begin{array}{cc}
\phi_{1}^{0} & \phi_{2}^{+} \\
\phi_{1}^{-} & -\phi_{2}^{0 *}
\end{array}\right], \quad \Delta_{L, R}=\left[\begin{array}{cc}
\Delta^{+} / \sqrt{2} & \Delta^{++} \\
\Delta^{0} & -\Delta^{+} / \sqrt{2}
\end{array}\right]_{L, R}
$$

The first stage of symmetry breaking down to the SM symmetry takes the following form [20]

$$
\left\langle\Delta_{L}^{0}\right\rangle=0, \quad\left\langle\Delta_{R}^{0}\right\rangle=v_{R}
$$

with $v_{R}$ giving masses to the heavy charged and neutral gauge bosons $W_{R}, Z_{R}$, right-handed neutrinos and all the scalars except for the usual Higgs doublet (the light doublet in the bi-doublet $\Phi$ ). Next, the neutral components of $\Phi$ develop vevs and break the SM symmetry down to $U(1)_{e m}$

$$
\langle\Phi\rangle=v \operatorname{diag}\left(\cos \beta,-\sin \beta e^{-i a}\right)
$$


where $v$ is real and positive and $\beta<\pi / 4,0<a<2 \pi$.

In turn, $\Delta_{L}$ develops a tiny induced vev $\left\langle\Delta_{L}\right\rangle \propto v^{2} / v_{R}$ [21] which leads to the type II seesaw source of neutrino mas [12].

Domain wall problem. It is well known that the spontaneous breaking of discrete symmetries produces domain walls which would under normal circumstances destroy the success of the standard big-bang cosmological model, leading to a notorious domain wall problem. The simplest way out would be inflation, but in the minimal model there is no candidate for an inflaton. There is also a possibility of symmetry non-restoration at high temperature [22] which could prevent a formation of domain walls [23], but that is questionable [24]. Fortunately, the possible LR symmetry breaking terms due to quantum gravity, even if suppressed by the Planck scale and thus almost negligible, suffice to get rid [25] of the domain walls early enough and not spoil the success of the BBN. For this reason, we believe that the domain wall problem can be safely ignored, at least until one has a predictive of quantum gravity effects, and we thus turn to physical consequences of the LR and gauge symmetry breaking.

Gauge bosons. Without assuming LR symmetry one gets for the $W_{R}, Z_{R}$ masses

$$
M_{W_{R}}^{2}=g_{R}^{2} v_{R}^{2}, \quad M_{Z_{R}}^{2}=2\left(g_{R}^{2}+g_{B-L}^{2}\right) v_{R}^{2}
$$

where $g_{R}$ and $g_{B-L}$ gauge couplings correspond to $S U(2)_{R}$ and $(B-L) / 2$, respectively. Hence a strict limit $M_{Z_{R}} \gtrsim \sqrt{2} M_{W_{R}}$, which guarantees that $W_{R}$ must be seen before $Z_{R}$.

For the LR symmetric gauge couplings relevant for the minimal model $g \equiv g_{L}=g_{R}$ one gets then a simplified expression

$$
M_{Z_{R}}=\sqrt{2} \frac{\cos \theta_{W}}{\sqrt{\cos 2 \theta_{W}}} M_{W_{R}} \simeq \sqrt{3} M_{W_{R}}
$$

The new neutral gauge boson $Z_{R}$ is substantially heavier that its charged counterpart $W_{R}$, which makes it unlikely to be discovered at the LHC (see more below).

Quark sector. The LR symmetry restricts severely quark Yukawa couplings. In the case of quarks the Yukawas are either hermitian for generalised parity or symmetric for generalised charge conjugation. In the latter case this guarantees the equality of left and right quark mixing angles, with five extra arbitrary phases in the RH current. The case of $P$ is more involved since the complex vev of (3.7) makes quark mass matrices non-hermitian. The search for the RH quark mixing matrix $V_{R}$ has been a great challenge for now forty years, and only recently the following analytic form valid in the entire parameter space was finally obtained [17].

Remarkably, in spite of near maximal LR symmetry breaking at low energies, the LH and RH mixing angles end up being almost exactly the same, while the new RH phases depend on a single parameter $s_{a} t_{2 \beta}$ which measures the departure from the hermiticity of quark mass matrices. One has the upper bound $s_{a} t_{2 \beta} \lesssim 2 m_{b} / m_{t}$ and the following expression for the quark mixing $V_{R}$ at first order [17]

$$
\left(V_{R}\right)_{i j} \simeq\left(V_{L}\right)_{i j}-i s_{a} t_{2 \beta}\left[\frac{\left(V_{L} m_{d} V_{L}^{\dagger}\right)_{i k}\left(V_{L}\right)_{k j}}{m_{u_{i}}+m_{u_{k}}}+\frac{\left(V_{L}\right)_{i k}\left(V_{L}^{\dagger} m_{u} V_{L}\right)_{k j}}{m_{d_{k}}+m_{d_{j}}}\right]+O\left(s_{a}^{2} t_{2 \beta}^{2}\right)
$$


The convergence is quite fast provided the $s_{a} t_{2 \beta}$ is not close to its upper limit; in any case it is straightforward to obtain higher order terms of the series [17]. As an illustration we give the difference between left and right mixing angles

$$
\begin{aligned}
& \theta_{R}^{12}-\theta_{L}^{12} \simeq-s_{a} t_{2 \beta} \frac{m_{t}}{m_{s}} \sin \theta_{L}^{23} \sin \theta_{L}^{13} \sin \delta_{L} \\
& \theta_{R}^{23}-\theta_{L}^{23} \simeq-s_{a} t_{2 \beta} \frac{m_{t}}{m_{b}} \frac{m_{s}}{m_{b}} \sin \theta_{L}^{12} \sin \theta_{L}^{13} \sin \delta_{L} \\
& \theta_{R}^{13}-\theta_{L}^{13} \simeq s_{a} t_{2 \beta} \frac{m_{t}}{m_{b}} \frac{m_{s}}{m_{b}} \sin \theta_{L}^{12} \sin \theta_{L}^{23} \sin \delta_{L}
\end{aligned}
$$

Since the LR symmetry in weak interactions is maximally broken at low energies, one would generally expect large differences of quark mixing angles. This would be true if not for the fact that the CKM mixing angles are small to start with, as manifest from (3.11), (3.12) and (3.13).

Leptonic sector. A question could be asked as what happens if the $N$ masses are small, so small that one ends up effectively with the case of Dirac neutrinos. It requires extremely small Dirac Yukawas for neutrinos, hard to achieve in the minimal model [26]. The seesaw mechanism emerged as a solution to this unappealing and un-natural scenario. In the seesaw picture the Majorana neutrino mass matrix is given by [21]

$$
M_{v}=M_{L}-M_{D}^{T} \frac{1}{M_{N}} M_{D}
$$

where $M_{D}$ is the neutrino Dirac mass matrix, while $M_{L} \propto M_{W_{L}}^{2} / M_{W_{R}}$ and $M_{N} \propto M_{W_{R}}$ are the symmetric Majorana mass matrices of left- and right-handed neutrinos, respectively. The smallness of neutrino mass is the consequence of near maximality of parity violation in beta decay, and in the infinite limit for the $W_{R}$ mass one recovers massless neutrinos of the SM.

The case of $C$ as the LR symmetry is rather illustrative, since it implies $M_{L}=v_{L} / v_{R} M_{N}$ and symmetric Dirac mass matrices $M_{D}=M_{D}^{T}$. The latter relation eliminates the arbitrary complex orthogonal matrix $O$ that obscures the usual seesaw mechanism in the SM with $N$. This provides the fundamental difference between the naive seesaw and the LR symmetric theory, since in LR Dirac mass matrix $M_{D}$ can be obtained directly from (3.14). In the type I seesaw picture, chosen only for the sake of simplicity, one gets [9]

$$
M_{D}=i M_{N} \sqrt{M_{N}^{-1} M_{V}}
$$

and thereby one can determine the mixing between light and heavy neutrinos. The square root in 3.15 has a number of discrete solutions and only in some pathological points continuous arbitrary parameters can arise.

The essential point is that the knowledge of $M_{D}$, or equivalently $Y_{D}$, allows for a direct probe of the Higgs origin of the neutrino mass, in analogy with the charged fermions in the SM. Namely, one can predict the associated Higgs, $W$ and $Z$ decays into $N$, or vice versa if $N$ are heavy enough. The latter case is illustrated in Fig.1 for two different values of the $W_{R}$ gauge boson mass. In the range of $N$ mass between $100-200 \mathrm{GeV}$ these decays becomes potentially observable at the LHC. 


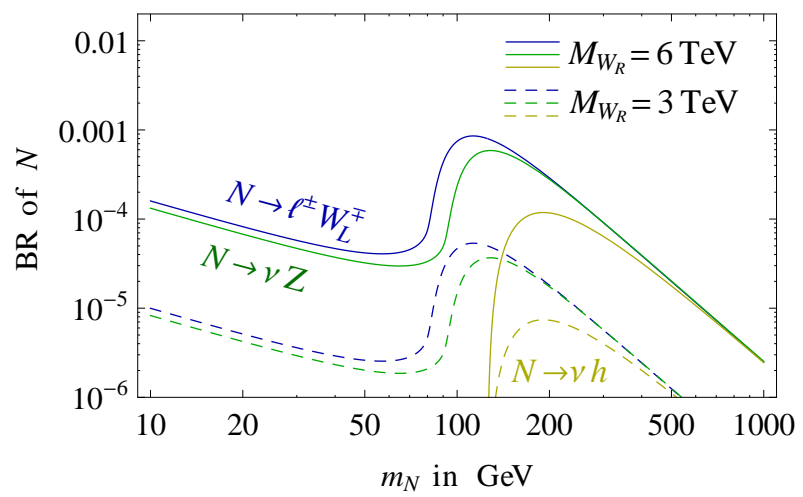

Figure 1: Branching ratio for the decay of heavy $N$ into the Higgs and SM gauge bosons, proceeding via Dirac couplings [9].

The situation in the case of $P$ is more subtle, but similar constraints emerge and again Dirac Yukawas get determined [42].

As we will see, if the scale of parity restoration is in the few $\mathrm{TeV}$ region, the theory offers a rich LHC phenomenology and a plethora of lepton flavor violating processes. Even more important, there is a deep connection between lepton number violation at LHC and in neutrinoless double decay [28].

The left and right-handed charged gauge bosons with their corresponding leptonic interactions in the mass eigenstate basis are

$$
\mathscr{L}_{W}=-\frac{g}{\sqrt{2}}\left(\bar{v}_{L} V_{L}^{\dagger} W_{L} e_{L}+\bar{N}_{R} V_{R}^{\dagger} W_{R} e_{R}\right)+\text { h.c. }
$$

In order to avoid cumbersome indices, we use the same notation for quark and lepton mixing matrices. We caution the reader not to confuse them.

Limits on the LR scale. The $K_{L}-K_{S}$ mass difference implies a lower theoretical limit on $M_{W_{R}}$ on the order of a few $\mathrm{TeV}$ [33]. The limit on the scale was sharpened in recent years in [34]. We will not discuss it here, since it was recently reviewed by us in [35]. Moreover, the LHC is slowly but surely catching up with theory; for a large range of $N$ masses one gets $M_{W_{R}} \gtrsim 3 \mathrm{TeV}$ [36].

LR symmetry and Grand unification. The minimal grand unified theory that contains the LR model is based on the $S O(10)$ gauge group. The important question is whether the LHC accessible scale can be incorporated in the case of the minimal model; and the answer is unfortunately no. This nice possibility existed before the weak mixing angle was measured precisely [37], but in the real world the LR scale must be quite large, on the order of $10^{10} \mathrm{GeV}$ or so [38]. Of course, one can always bring the scale down by increasing the number of Higgs multiplets, but then one loses the predictions of grand unification and thus to us that is more semantics than physics. Grand unification is a deep and beautiful idea but the picture of the desert is rather painful, especially today that we LHC on our hands and new accelerators thought about seriously. 
LR symmetry and naturalness. A lover of naturalness must be worried at this point since clearly this theory is plagued by the same hierarchy issue as the SM. One can always resort to low energy supersymmetry as a way to naturalness and clearly one did. The supersymmetric version of the LR model has been studied extensively over the years (one of us spent a great deal of time working on it), but here we prefer to restrict ourselves to the minimal model in order to boost predictivity and simplicity. Suffice to say that for the low LR scale R-parity must be broken [39] whereas in the high scale case one can show that it remains exact [40]

\subsection{Lepton Number Violation}

The Majorana nature of $v$ and $N$ implies Lepton Number Violation (LNV), both at low and high energies and we discuss here some important examples.

Neutrinoless double beta decay. This low energy process is the text-book example of LNV. In this theory it is induced by the usual exchange of light Majorana neutrinos and the $W$ boson, and also by its RH analog exchange of $N$ and $W_{R}$ as noticed already in [6]. The new contribution can easily dominate the usual neutrino one, and moreover, if neutrino masses turn out to be small, it may be necessary in case this process gets observed in near future. If that were to happen, the $W_{R}$ mass would have to lie tantalisingly close to the LHC reach [28].

The total effective mass parameter controlling the rate of neutrinoless double beta decay due to light and heavy neutrinos is given by

$$
\left|m_{v+N}^{e e}\right|=\sqrt{\left|\left(M_{V}\right)_{e e}\right|^{2}+\frac{\left|\left(V_{R}\right)_{11}^{q}\right|^{4}}{\left|\left(V_{L}\right)_{11}^{q}\right|^{4}} \frac{M_{W_{L}}^{8}}{M_{W_{R}}^{8}}\left|\left(\frac{k^{2}}{M_{N}}\right)_{e e}\right|^{2}}
$$

where $M_{v}$ and $M_{N}$ are the corresponding neutrino mass matrices, $k$ is a measure of the light neutrino momentum on the order of $100 \mathrm{MeV}$ and depends on the specific nucleus, and $V_{L, R}^{q}$ are the left and right-handed quark mixing matrices. The knowledge of $V_{R}^{q}$ is essential in order to make accurate calculation of the decay rate. From (3.10) it is easily shown that $\left|\left(V_{R}\right)_{11}^{q}\right| /\left|\left(V_{L}\right)_{11}^{q}\right| \simeq 1$ which simplifies the analysis.

An illustration requires the knowledge of the right-handed leptonic mixing matrix. A simple example is provided by the type II seesaw as shown in Fig. 2. In this case the usual cancellation which occurs in the light neutrino exchange of normal spectrum disappears and both hierarchies become equally promising.

LHC signatures. The Majorana nature of $N$ allows also for the direct LNV at hadronic colliders: the Keung-Senjanovic (KS) production process of two same signs charged leptons accompanied with two jets [8], shown in the Fig. 3. Moreover this process allows for the possibility of establishing directly the Majorana nature of $N$ since then both same and opposite sign charged leptons decay products occur with the same probability. It should be stressed that this has become the paradigm for LNV at the hadronic colliders, and it occurs in basically any theory that leads to Majorana neutrinos.

From the KS process one could probe both $W_{R}$ and $N$ masses, and also the RH leptonic mixing angles $[43,44]$. The detailed studies for the LHC were performed in $[45,46]$ where it was argued that high luminosity of $300 \mathrm{fb}^{-1}$ reach goes all the way to $5-6 \mathrm{TeV}$ (for a roadmap at the LHC, see 


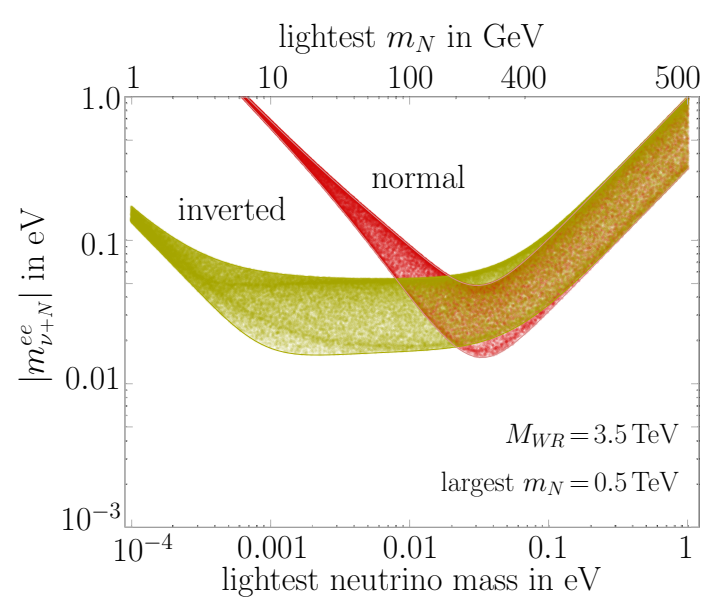

Figure 2: The total contribution of light and heavy neutrino masses to neutrinoless double beta decay effective mass parameter.

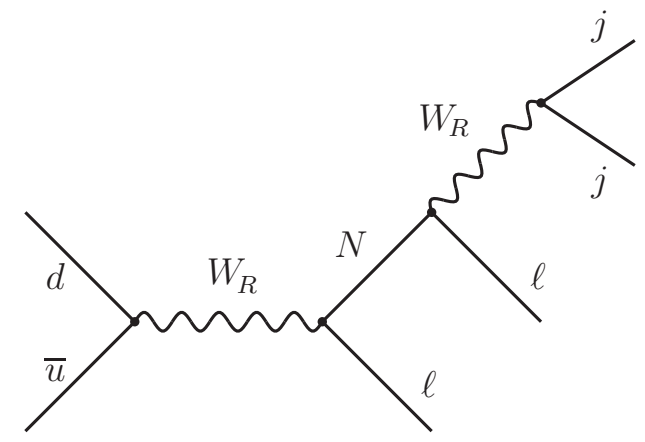

Figure 3: The KS production process of lepton number violating same sign di-leptons through the production and subsequent decay of $N$.

[47]). Moreover, one could measure the chirality of $N$ couplings and establish their RH nature [45, 48]. For reviews of this subject, see [49].

In the LR model the dominant LNV effect is through the on-shell production of $W_{R}$; it could also occur through the small $v-N$ mixing and the usual $W$ exchange, but that requires huge $M_{D}$ [50].

What happens with the LNV when $W_{R}$ is too heavy to be observed directly? Since $W_{R}$ mixes with $W_{L}$ one could hope for indirect effects due to the mixing, but it is too small $\left(\theta_{L R} \lesssim 10^{-3}\right)$ to matter at the LHC. We could still imagine the doubly charged scalars in $\Delta L, R$ to be light enough to see LNV through their decays into lepton pairs, but what if even they were too heavy to be seen? One last hope is provided by the SM Higgs boson decays as long it mixes appreciably with the neutral $\Delta_{R}$ scalar, since in this case one can obtain LNV $h \rightarrow N N$ decays [51], with $\mathrm{N}$ decaying into a charged lepton and two jets. This has been recently revisited in the context of the LHC and argued in favor of its feasibility [52]. 


\subsection{Lepton Flavor Violation}

Low energy signatures. There are a number of LFV processes providing constraints on the masses and gauge mixings of right-handed neutrinos and on the masses of the doubly charged scalars, such as $\mu-e$ conversion in nuclei, $\mu \rightarrow e \gamma, \mu \rightarrow 3 e$, and their analogues for the $\tau$ lepton, rare $K$ and $B$ meson decays, and so on.

The most relevant constraint up to date arises from the muon rare decay $\mu \rightarrow 3 e$ with an experimental limit $\operatorname{BR}(\mu \rightarrow 3 e)<1.0 \times 10^{-12}$ [?]. The branching ratio induced by the tree level exchange of doubly charged bosons $\Delta_{L}^{++}$and $\Delta_{R}^{++}$is given by

$$
\operatorname{BR}(\mu \rightarrow 3 e)=\frac{1}{2}\left(\frac{M_{W}}{M_{W_{R}}}\right)^{4}\left|V_{R} \frac{m_{N}}{m_{\Delta}} V_{R}^{T}\right|_{e \mu}^{2}\left|V_{R} \frac{m_{N}}{m_{\Delta}} V_{R}^{T}\right|_{e e}^{2}
$$

where $1 / m_{\Delta}^{2} \equiv 1 / m_{\Delta_{L}}^{2}+1 / m_{\Delta_{R}}^{2}$ and $V_{R}$ stands for the right-handed leptonic mixing matrix. For light $W_{R}$ gauge boson mass at the TeV scale, the LFV rates are mainly controlled by the ratio $m_{N} / m_{\Delta}$, in addition to mixing angles and phases. Roughly one has the upper bound $m_{N} / m_{\Delta} \lesssim 0.1$. The Fig. 4 exemplifies the upper limit in the case of type II seesaw.
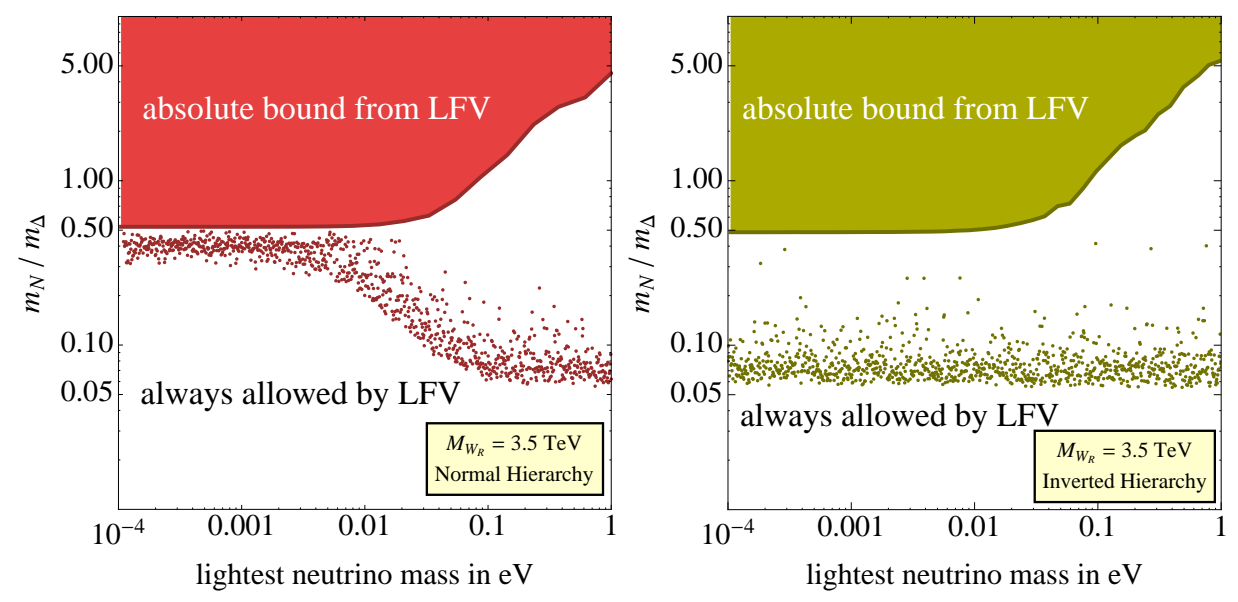

Figure 4: Upper bounds on $m_{N}^{\text {heaviest }} / m_{\Delta}$ from $\mu \rightarrow 3 e$ for a representative value of $M_{W_{R}}=3.5 \mathrm{TeV}$. The dots show the (most probable) upper bounds resulting for different mixing angles and phases whereas the dark line represent the absolute upper bound.

High energy signatures. We have already remarked in the previous subsection that the KS process allows for the determination of the RH leptonic mixing matrix. This includes all possible LVF channels which may or may not be LNV processes.

As in the low energy case, the production of decay of the doubly charged bosons $\Delta_{L}^{++}$and $\Delta_{R}^{++}$leads in general to LFV and can be used to extract the RH leptonic mixings [44]. This is reminiscence of the type II seesaw we commented on at the beginning.

\subsection{Dark matter}

It can be easily shown that the only candidate for the DM particle in the MLRSM is the lightest RH neutrino if it is light enough to be sufficiently stable. For this reason it cannot act as the cold 
$\mathrm{DM}$, but it could be a warm DM with a mass around $\mathrm{keV}[53,54]$. This is reminiscent of the original SM case augmented with N's [55], so it works for a heavy $W_{R}$ as expected. However, there is a possible window for $M_{W_{R}} \simeq 5 \mathrm{GeV}$, accessible at the LHC. What makes it really interesting is that the spectrum and mixings of RH neutrinos get completely fixed, but unfortunately they end up too light to be observed in the KS process, thus making it hard to probe directly the Higgs origin of neutrino mass.

Speaking strictly for ourselves, we would prefer a different DM candidate although that requires going beyond the minimal model (unless DM is in the form of microscopic black holes). At this point we reserve our judgment, and do not insist on the above DM scenario. If $W_{R}$ were really light enough to be observed at the LHC, it would be rather exciting to see what the RH neutrino mass spectrum is.

\section{Other theories of neutrino mass}

MSSM and neutrino mass. A well motivated example is the Minimal Supersymmetric Standard Model (MSSM) since in its generality it predicts non-vanishing neutrino mass and even the seesaw mechanism. If one does not assume R-parity conservation (R-parity is completely ad-hoc in the MSSM), one has massive Majorana neutrino and the associated rich physics both in the neutrinoless double beta decay and at the LHC [56]. The lightest neutrino then cannot play the role of the dark matter, but there is always gravitino as a natural DM candidate due to its longevity. Unfortunately, a proliferation of parameters due to the ignorance of sfermion masses and mixings prevents one from making any predictions without making arbitrary assumptions. For this reason we did not discuss it here. We only make a passing remark, important for the fans of the high scale seesaw mechanism. In this case, as long as the seesaw is based on the gauged B-L [57] as in LR symmetry, Pati-Salam and $S O(10)$, Rp remains exact at all energies [58].

Grand unification and neutrino mass probe at the LHC . What about grand unification and the probe of neutrino mass? While $\mathrm{SO}(10)$ theory is the most natural candidate as a theory of neutrino mass, in the minimal versions the scale of new physics is not directly accessible so it is logical to turn to the original prototype of grand unification, the $S U(5)$ theory. The minimal model of Georgi and Glashow [32] was remarkably predictive, so much that it managed to fail in spite of possible threshold effects at the GUT scale. It fails doubly: (i) it does not unify and (ii) just as the SM, it predicts massless neutrino, The simplest way to cure (i) is to add an adjoint fermion representation [10], for it has gaugino like particles, essential for the success of the low energy supersymmetric unification. This theory then leads to a hybrid hybrid type I plus type III seesaw, and most important it predicts a light weak triplet fermion [10] with a mass in the TeV energy range. Its decays allow one to reconstruct the neutrino mass matrix, for it turns out that the lightest neutrino is effectively massless [16]. A careful study [59] shows that the triplet can be observed at the $14 \mathrm{TeV}$ LHC with $100 \mathrm{fb}^{-1}$ luminosity with the mass up to $700 \mathrm{GeV}$.

\section{Neutrino mass and the interpretation of recent excesses at the LHC.}

There have been a number of reported excesses recently at the LHC with different degrees of certainty (around a few sigma), but none close to be discoveries [60]. Some interesting channels 
are of $W Z, W h$ and $e \bar{e} j j$ type and could be in principle explained through the production of $W_{R}$ and its decays, as long as the LH and RH gauge couplings are not the same. The $e \bar{e} j j$ process is not accompanied by the LNV eejj as in the case of the KS, which requires the degenerate Majorana RH neutrinos in the minimal LR model [61]. There is also a di-photon decay excess [62] around $3 \sigma$ of a supposed neutral $750 \mathrm{GeV}$ resonance which produced a flurry of activity in recent weeks. In the meantime there is a claim that the actual significance is lower, more like $\sigma$ [63]. While the minimal LR possesses neutral scalars such as $\delta_{L, R}^{0}$ that can decay into two photons through a $W_{R}$ and charged Higgs loop, the cross section is not in accord with the data [64].

We personally believe, however, and bet that all of these anomalies will go away and so we ignore them here.

\section{Summary and Outlook}

We discussed here an experimental probe of Majorana neutrino mass origin, both at colliders through the production of the same sign di-leptons, and through neutrinoless double beta decay. A classical example is provided by the LR symmetric theory that predicts the existence of righthanded neutrinos and leads to the seesaw mechanism. A TeV scale LR symmetry would have spectacular signatures at LHC, with a possible discovery of $W_{R}$ and $v_{R}$. This offers a possibility of observing parity restoration and the Majorana nature of neutrinos. Furthermore, the measurements at the colliders can fix the masses and the mixings of the right-handed neutrinos, which in turn can make predictions for the neutrinoless double beta decay and lepton flavor violation [28].

One of the main messages that we wish to convey is that, contrary to the conventional claims in the literature, neutrinoless double beta decay may be dominated by new physics and not by neutrino masses. This would be great news for if new physics were a source of the neutrinoless double beta decay it would have to lie at the $\mathrm{TeV}$ scale in order to provide a large enough effect. In other words, new physics behind neutrinoless double beta decay is likely to be at the LHC reach.

In summary, we hope to have convinced you that the LHC has all the potential to probe the origin of neutrino mass, and complete the picture of the Higgs mechanism behind elementary particle masses.

\section{Acknowledgements}

G.S. wishes to thank the organisers of the Planck 2015 for the opportunity to present this material in the proceedings in spite of being unable to participate due to unfortunate circumstances. We are grateful to our collaborators on the issues discussed here, notably Wai-Yee Keung, Rabi Mohapatra, Alessio Maiezza, Miha Nemevsek, Fabrizio Nesti and Yue Zhang.

\section{References}

[1] S. Weinberg, “A Model of Leptons,” Phys. Rev. Lett. 19, 1264 (1967).

[2] J. C. Pati and A. Salam, "Lepton Number As The Fourth Color," Phys. Rev. D 10 (1974) 275.

R. N. Mohapatra and J. C. Pati, “A 'Natural' Left-Right Symmetry,” Phys. Rev. D 11 (1975) 2558. 
G. Senjanović and R. N. Mohapatra, "Exact Left-Right Symmetry And Spontaneous Violation Of Parity,” Phys. Rev. D 12 (1975) 1502.

G. Senjanović, "Spontaneous Breakdown Of Parity In A Class Of Gauge Theories," Nucl. Phys. B 153 (1979) 334.

[3] P. Minkowski, "Mu $\rightarrow$ E Gamma At A Rate Of One Out Of 1-Billion Muon Decays?,” Phys. Lett. B 67 (1977) 421.

R. N. Mohapatra and G. Senjanović, "Neutrino Mass and Spontaneous Parity Violation," Phys. Rev. Lett. 44 (1980) 912.

S. L. Glashow, “The Future of Elementary Particle Physics,” NATO Sci. Ser. B 61, 687 (1980).

M. Gell-Mann, P. Ramond and R. Slansky, "Complex Spinors and Unified Theories," Conf. Proc. C 790927 (1979) 315 [arXiv:1306.4669 [hep-th]].

T. Yanagida, "Horizontal Symmetry And Masses Of Neutrinos," Conf. Proc. C 7902131, 95 (1979).

[4] G. Racah, "On the symmetry of particle and antiparticle," Nuovo Cim. 14, 322 (1937)

W. H. Furry, “On transition probabilities in double beta-disintegration," Phys. Rev. 56, 1184 (1939).

[5] E. Majorana, "Theory of the Symmetry of Electrons and Positrons," N. Cim. 14 (1937) 171.

[6] R. N. Mohapatra and G. Senjanović, "Neutrino Mass and Spontaneous Parity Violation," Phys. Rev. Lett. 44 (1980) 912.

[7] G. Feinberg, M. Goldhaber, Proc. Nat. Ac. Sci. USA 45 (1959) 1301;

B. Pontecorvo, "Superweak interactions and double beta decay," Phys. Lett. B26 (1968) 630.

[8] W. Y. Keung and G. Senjanović, "Majorana Neutrinos And The Production Of The Right-Handed Charged Gauge Boson,” Phys. Rev. Lett. 50, 1427 (1983).

[9] M. Nemevšek, G. Senjanović and V. Tello, "Connecting Dirac and Majorana Neutrino Mass Matrices in the Minimal Left-Right Symmetric Model," Phys. Rev. Lett. 110 (2013) 15, 151802 [arXiv:1211.2837 [hep-ph]].

[10] B. Bajc and G. Senjanović, “Seesaw at LHC," JHEP 0708, 014 (2007) [arXiv:hep-ph/0612029].

[11] S. Weinberg, "Baryon And Lepton Nonconserving Processes," Phys. Rev. Lett. 43, 1566 (1979).

[12] M. Magg and C. Wetterich, "Neutrino Mass Problem And Gauge Hierarchy,” Phys. Lett. B 94 (1980) 61.

G. Lazarides, Q. Shafi and C. Wetterich, "Proton Lifetime And Fermion Masses In An SO(10) Model,” Nucl. Phys. B 181 (1981) 287.

R. Mohapatra, G. Senjanović, "Neutrino Masses And Mixings In Gauge Models With Spontaneous Parity Violation,” Phys.Rev.D23 (1981) 165.

[13] R. Foot, H. Lew, X. G. He and G. C. Joshi, "Seesaw Neutrino Masses Induced by a Triplet of Leptons,” Z. Phys. C 44, 441 (1989).

[14] J. A. Casas and A. Ibarra, "Oscillating neutrinos and muon $\rightarrow$ e, gamma," Nucl. Phys. B 618, 171 (2001) [hep-ph/0103065].

[15] J. Garayoa and T. Schwetz, "Neutrino mass hierarchy and Majorana CP phases within the Higgs triplet model at the LHC," JHEP 0803 (2008) 009 [arXiv:0712.1453 [hep-ph]].

M. Kadastik, M. Raidal and L. Rebane, "Direct determination of neutrino mass parameters at future colliders," Phys. Rev. D 77, 115023 (2008) [arXiv:0712.3912 [hep-ph]]. 
P. Fileviez Pérez, T. Han, G. Y. Huang, T. Li and K. Wang, "Testing a Neutrino Mass Generation Mechanism at the Large Hadron Collider," Phys. Rev. D 78, 071301 (2008) [arXiv:0803.3450 [hep-ph]].

[16] B. Bajc, M. Nemevšek and G. Senjanović, “Probing seesaw at LHC,” Phys. Rev. D 76, 055011 (2007) [arXiv:hep-ph/0703080].

[17] G. Senjanović and V. Tello, "Right Handed Quark Mixing in Left-Right Symmetric Theory," Phys. Rev. Lett. 114, no. 7, 071801 (2015) [arXiv:1408.3835 [hep-ph]].

G. Senjanović and V. Tello, "Restoration of Parity and the Right-Handed Analog of the CKM Matrix," arXiv:1502.05704 [hep-ph].

[18] T. D. Lee, C. -N. Yang, Phys. Rev. 104, 254-258 (1956).

[19] Minkowski, Ref. [3]

Mohapatra, Senjanović, Ref. [3]

[20] G. Senjanović and R. N. Mohapatra, "Exact Left-Right Symmetry and Spontaneous Violation of Parity," Phys. Rev. D 12, 1502 (1975).

G. Senjanović, "Spontaneous Breakdown Of Parity In A Class Of Gauge Theories," Nucl. Phys. B 153 (1979) 334.

R. Mohapatra, G. Senjanović, in [12]

[21] R. Mohapatra, G. Senjanović, in [12]

[22] S. Weinberg, "Gauge and Global Symmetries at High Temperature," Phys. Rev. D 9 (1974) 3357.

R. N. Mohapatra and G. Senjanović, "Broken Symmetries at High Temperature," Phys. Rev. D 20, 3390 (1979).

R. N. Mohapatra and G. Senjanović, "High Temperature Behavior of Gauge Theories," Phys. Lett. B 89, 57 (1979).

[23] G. R. Dvali, G. Senjanović, "Is there a domain wall problem?,” Phys. Rev. Lett. 74 (1995) 5178-5181. [hep-ph/9501387].

G. R. Dvali, A. Melfo, G. Senjanović, "Nonrestoration of spontaneously broken P and CP at high temperature," Phys. Rev. D54, 7857-7866 (1996). [hep-ph/9601376].

[24] G. Bimonte and G. Lozano, "On Symmetry nonrestoration at high temperature," Phys. Lett. B 366, 248 (1996) [hep-th/9507079].

[25] B. Rai, G. Senjanović, “Gravity and domain wall problem,” Phys. Rev. D49, 2729-2733 (1994). [hep-ph/9301240].

[26] G. C. Branco and G. Senjanović, "The Question of Neutrino Mass," Phys. Rev. D 18 (1978) 1621.

[27] M. Nemevšek, G. Senjanović and V. Tello, to appear.

[28] V. Tello, M. Nemevsek, F. Nesti, G. Senjanovic and F. Vissani, "Left-Right Symmetry: from LHC to Neutrinoless Double Beta Decay," Phys. Rev. Lett. 106 (2011) 151801 doi:10.1103/PhysRevLett.106.151801 [arXiv:1011.3522 [hep-ph]].

M. Nemevšek, F. Nesti, G. Senjanović and V. Tello, "Neutrinoless Double Beta Decay: Low Left-Right Symmetry Scale?," arXiv:1112.3061 [hep-ph].

[29] Minkowski, Ref. [3]. 
[30] F. Vissani, "Signal of neutrinoless double beta decay, neutrino spectrum and oscillation scenarios," JHEP 9906, 022 (1999). [hep-ph/9906525].

[31] A. Ferrari et al., "Sensitivity study for new gauge bosons and right-handed Majorana neutrinos in $\mathrm{p} \mathrm{p}$ collisions at s = 14-TeV,” Phys. Rev. D 62, 013001 (2000).

S. N. Gninenko, M. M. Kirsanov, N. V. Krasnikov and V. A. Matveev, "Detection of heavy Majorana neutrinos and right-handed bosons," Phys. Atom. Nucl. 70, 441 (2007).

[32] H. Georgi and S. L. Glashow, "Unity Of All Elementary Particle Forces,” Phys. Rev. Lett. 32, 438 (1974).

[33] G. Beall, M. Bander and A. Soni, "Constraint on the Mass Scale of a Left-Right Symmetric Electroweak Theory from the K(L) K(S) Mass Difference,” Phys. Rev. Lett. 48, 848 (1982).

R. N. Mohapatra, G. Senjanović and M. D. Tran, "Strangeness Changing Processes and the Limit on the Right-handed Gauge Boson Mass,” Phys. Rev. D 28, 546 (1983).

[34] Y. Zhang, H. An, X. Ji and R. N. Mohapatra, "General CP Violation in Minimal Left-Right Symmetric Model and Constraints on the Right-Handed Scale,” Nucl. Phys. B 802, 247 (2008) [arXiv:0712.4218 [hep-ph]].

F. Xu, H. An and X. Ji, "Neutron Electric Dipole Moment Constraint on Scale of Minimal Left-Right Symmetric Model,” JHEP 1003, 088 (2010) [arXiv:0910.2265 [hep-ph]].

A. Maiezza, M. Nemevšek, F. Nesti, G. Senjanović, “Left-Right Symmetry at LHC," Phys. Rev. D82 (2010) 055022. [arXiv:1005.5160 [hep-ph]].

M. Blanke, A. J. Buras, K. Gemmler and T. Heidsieck, JHEP 1203 (2012) 024 [arXiv:1111.5014 [hep-ph]].

S. Bertolini, A. Maiezza and F. Nesti, "Present and Future K and B Meson Mixing Constraints on TeV Scale Left-Right Symmetry,” Phys. Rev. D 89, no. 9, 095028 (2014) [arXiv:1403.7112 [hep-ph]].

A. Maiezza and M. Nemevšek, "Strong P invariance, neutron electric dipole moment, and minimal left-right parity at LHC,” Phys. Rev. D 90, no. 9, 095002 (2014) [arXiv:1407.3678 [hep-ph]].

[35] S. Alekhin et al., arXiv:1504.04855 [hep-ph].

[36] V. Khachatryan et al. [CMS Collaboration], "Search for heavy neutrinos and W bosons with right-handed couplings in proton-proton collisions at sqrt(s) = $8 \mathrm{TeV}$," arXiv:1407.3683 [hep-ex].

[37] T. G. Rizzo and G. Senjanović, "Can There Be Low Intermediate Mass Scales in Grand Unified Theories?,” Phys. Rev. Lett. 46 (1981) 1315. doi:10.1103/PhysRevLett.46.1315

[38] T. G. Rizzo and G. Senjanović, "Grand Unification and Parity Restoration at Low-energies. 2. Unification Constraints,” Phys. Rev. D 25 (1982) 235. doi:10.1103/PhysRevD.25.235

[39] R. Kuchimanchi and R. N. Mohapatra, “No parity violation without R-parity violation,” Phys. Rev. D 48, 4352 (1993) doi:10.1103/PhysRevD.48.4352 [hep-ph/9306290].

[40] C. S. Aulakh, A. Melfo and G. Senjanović, "Minimal supersymmetric left-right model," Phys. Rev. D 57 (1998) 4174 doi:10.1103/PhysRevD.57.4174 [hep-ph/9707256].

[41] G. Senjanović and P. Senjanović, "Suppression of Higgs Strangeness Changing Neutral Currents in a Class of Gauge Theories," Phys. Rev. D 21 (1980) 3253.

[42] M. Nemevšek, G. Senjanović and V. Tello, to appear.

[43] S. P. Das, F. F. Deppisch, O. Kittel and J. W. F. Valle, "Heavy Neutrinos and Lepton Flavour Violation in Left-Right Symmetric Models at the LHC," Phys. Rev. D 86, 055006 (2012) [arXiv:1206.0256 [hep-ph]]. 
[44] J. C. Vasquez, "Right-handed lepton mixings at the LHC," arXiv:1411.5824 [hep-ph].

[45] A. Ferrari et al., "Sensitivity study for new gauge bosons and right-handed Majorana neutrinos in $p p$ collisions at $s=14-\mathrm{TeV}$," Phys. Rev. D 62 (2000) 013001.

[46] S. N. Gninenko, M. M. Kirsanov, N. V. Krasnikov and V. A. Matveev, "Detection of heavy Majorana neutrinos and right-handed bosons," Phys. Atom. Nucl. 70 (2007) 441.

[47] M. Nemevšek, F. Nesti, G. Senjanović and Y. Zhang, "First Limits on Left-Right Symmetry Scale from LHC Data," Phys. Rev. D 83, 115014 (2011) [arXiv:1103.1627 [hep-ph]].

[48] T. Han, I. Lewis, R. Ruiz and Z. g. Si, "Lepton Number Violation and $W^{\prime}$ Chiral Couplings at the LHC,” Phys. Rev. D 87, 035011 (2013) [Erratum-ibid. D 87, no. 3, 039906 (2013)] [arXiv:1211.6447 [hep-ph]].

[49] G. Senjanović, "Seesaw at LHC through Left - Right Symmetry," Int. J. Mod. Phys. A 26, 1469 (2011) [arXiv:1012.4104 [hep-ph]].

G. Senjanović, "Neutrino mass: From LHC to grand unification," Riv. Nuovo Cim. 034, 1 (2011). V. Tello, PhD Thesis, SISSA (2012) "Connections between the high and low energy violation of Lepton and Flavor numbers in the minimal left-right symmetric model,"

[50] A. Pilaftsis, "Radiatively induced neutrino masses and large Higgs neutrino couplings in the standard model with Majorana fields," Z. Phys. C 55 (1992) 275 [hep-ph/9901206].

A. Datta, M. Guchait and A. Pilaftsis, "Probing lepton number violation via majorana neutrinos at hadron supercolliders," Phys. Rev. D 50, 3195 (1994) [hep-ph/9311257].

[51] J. F. Gunion, H. E. Haber, G. L. Kane and S. Dawson, “The Higgs Hunter's Guide," Front. Phys. 80 (2000) 1.

[52] A. Maiezza, M. Nemev?ek and F. Nesti, "Lepton Number Violation in Higgs Decay at LHC," Phys. Rev. Lett. 115, 081802 (2015) doi:10.1103/PhysRevLett.115.081802 [arXiv:1503.06834 [hep-ph]].

[53] F. Bezrukov, H. Hettmansperger and M. Lindner, "keV sterile neutrino Dark Matter in gauge extensions of the Standard Model," Phys. Rev. D 81, 085032 (2010) [arXiv:0912.4415 [hep-ph]].

[54] M. Nemevšek, G. Senjanović and Y. Zhang, "Warm Dark Matter in Low Scale Left-Right Theory," JCAP 1207, 006 (2012) [arXiv:1205.0844 [hep-ph]].

[55] S. Dodelson and L. M. Widrow, "Sterile-neutrinos as dark matter," Phys. Rev. Lett. 72, 17 (1994) doi:10.1103/PhysRevLett.72.17 [hep-ph/9303287].

[56] B. C. Allanach, C. H. Kom and H. Pas, "Large Hadron Collider probe of supersymmetric neutrinoless double beta decay mechanism," Phys. Rev. Lett. 103 (2009) 091801 [arXiv:0902.4697 [hep-ph]].

B. C. Allanach, C. H. Kom, H. Pas, "LHC and B physics probes of neutrinoless double beta decay in supersymmetry without R-parity," JHEP 0910, 026 (2009). [arXiv:0903.0347 [hep-ph]].

[57] R. N. Mohapatra, "New Contributions to Neutrinoless Double beta Decay in Supersymmetric Theories," Phys. Rev. D 34, 3457 (1986).

[58] C. S. Aulakh, A. Melfo, A. Rašsin and G. Senjanović, "Seesaw and supersymmetry or exact R-parity," Phys. Lett. B 459, 557 (1999) [hep-ph/9902409].

[59] A. Arhrib, B. Bajc, D. K. Ghosh, T. Han, G. Y. Huang, I. Puljak and G. Senjanović, "Collider Signatures for Heavy Lepton Triplet in Type I+III Seesaw,” Phys. Rev. D 82 (2010) 053004 [arXiv:0904.2390 [hep-ph]]. 
[60] For a recent discussion and further references, see e.g. F. Dias, S. Gadatsch, M. Gouzevich, C. Leonidopoulos, S. Novaes, A. Oliveira, M. Pierini and T. Tomei, "Combination of Run-1 Exotic Searches in Diboson Final States at the LHC,” arXiv:1512.03371 [hep-ph].

[61] J. Gluza and T. Jelinski, "Heavy neutrinos and the $p p \rightarrow l l j j$ CMS data," Phys. Lett. B 748 (2015) 125 [arXiv:1504.05568 [hep-ph]].

[62] "ATLAS and CMS physics results from Run 2", talks by J. Olsen and M. Kado, CERN, December 15, 2015.

CMS Collaboration [CMS Collaboration], collisions at 13TeV,Ó CMS-PAS-EXO-15-004.

The ATLAS collaboration, ATLAS-CONF-2015-081.

[63] J. H. Davis, M. Fairbairn, J. Heal and P. Tunney, "The Significance of the 750 GeV Fluctuation in the ATLAS Run 2 Diphoton Data,” arXiv:1601.03153 [hep-ph].

[64] A. Dasgupta, M. Mitra and D. Borah, "Minimal Left-Right Symmetry Confronted with the $750 \mathrm{GeV}$ Di-photon Excess at LHC," arXiv:1512.09202 [hep-ph]. 